\title{
Determination of an adjusting rule in the case of multi-criteria inertial piloting
}

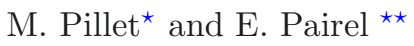 \\ Université de Savoie, Laboratoire SYMME, Annecy, France
}

Received: 21 February 2011 / Accepted: 3 March 2011

\begin{abstract}
The weighted inertial piloting of processes presented in this paper is a break-away in terms of the quality of machined mechanical workpieces compared to the traditional piloting carried out by machine setters. However, sometimes one has a stability problem during the piloting phase due to over adjustment. We propose to study the causes of this instability and to develop an approach in order to correct the problems. Once we have reminded ourselves of the basics of weighted inertial tolerancing, we will propose an approach to identify the most probable decentring. The applications of the concepts discussed in this article were tested over a period of two months. The results are very convincing, especially with the elimination of the phenomenon previously observed in identical conditions.
\end{abstract}

Keywords: Inertial tolerancing; inertial piloting; process adjusting; statistical process control; adjusting rules

\section{Introduction}

Inertial tolerancing ${ }^{1}[1-5]$ offers a new way to tackle the issue of product conformity. The objective is no longer to obtain the products within a given specification interval, but to minimise the variance around the target.

In order to meet this requirement in the case of the machining of mechanical workpiece, it is often necessary to look closely at the way the machines are set. Many adjusting approaches have been proposed using inertial control charts $[6,7]$, but the limits to these approaches are exposed in the case of multi-criteria processes when the characteristics are inter-dependent.

The use of adjusting matrices [8-10] and of weighted inertial adjusting [7] offers solutions which have brought a real breakthrough in terms of the quality of the products.

However despite these approaches, instability can still sometimes be observed and this results in the stratified distribution of products. This stratification comes from over-adjusting the production means. The objective of this article is to propose an approach which completes weighted inertial tolerancing by looking for the most probable decentring in order to avoid getting this phenomenon of stratification.

After having recalled the basic concepts of inertial tolerancing and of weighted inertial adjusting, we will now show that the most probable adjusting is not the decen-

\footnotetext{
${ }^{\star}$ Correspondence: Maurice.pillet@univ-savoie.fr

${ }^{\star \star}$ Correspondence: Eric.pairel@univ-savoie.fr

1 This work was supported by the European program INTERREG III France-Suisse and by the pole of competitiveness ARVE INDUSTRIE Mont-Blanc.
}

tring observed and we will offer a solution to calculate it. An example of weighted inertial adjusting using the most probable adjusting is given at the end of the article.

\section{Multi-criteria inertial adjusting}

\subsection{Multi-criteria inertial adjusting}

The objective of multi-criteria inertial adjusting is to minimise the inertia on the set of dependent characteristics in a process. It is particularly easy to use when machining using numerical control.

Let us recall that the inertia is calculated using the following equation (1):

$$
I=\sqrt{\sigma^{2}+\delta^{2}}
$$

with

$\sigma$ : the standard deviation of the population;

$\delta$ : the decentring in relation to the target.

The inertial conformity is defined by the XP E04 008 standard. A characteristic marked as $10^{0.01}$ STI means that the maximum inertia is equal to 0.01 . We illustrate this in the example given in Figure 1. We must guarantee the inertia on the characteristics $a$ to $e$ on this workpiece. Table 1 gives the target values and the different inertia of these characteristics.

The example in Figure 1 illustrates the mechanical problem well when several characteristics on the workpiece depend on different adjusting criteria. In the example, we 


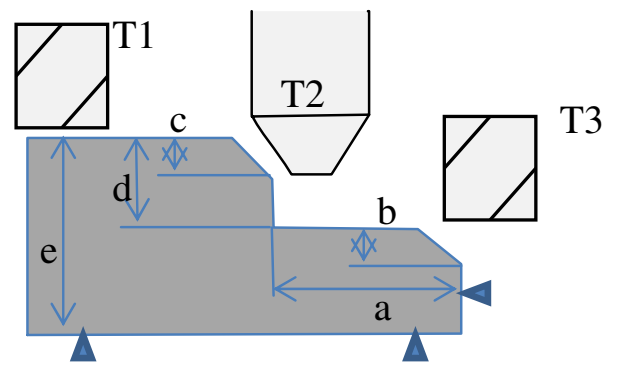

Fig. 1. Example of a workpiece to make using machining.

Table 1. Inertial tolerances.

\begin{tabular}{ccc}
\hline Characteristic & Target & $I_{M A X}$ \\
\hline$a$ & 5 & 0.003 \\
$b$ & 1 & 0.007 \\
$c$ & 1 & 0.007 \\
$d$ & 3 & 0.003 \\
$e$ & 6 & 0.017 \\
\hline
\end{tabular}

Table 2. Matrix $A$.

\begin{tabular}{ccccc}
\hline Characteristic & CT1L & CT2L & CT3L & CT3R \\
\hline$a$ & 0 & 0 & 0 & -1 \\
$b$ & 0 & -1 & 1 & 0 \\
$c$ & 1 & -1 & 0 & 1 \\
$d$ & 1 & 0 & -1 & 0 \\
$e$ & 1 & 0 & 0 & 0 \\
\hline
\end{tabular}

can easily find the matrix relation $E=A C$ which links the vector of the deviation vector to the target value of the characteristics $(E)$ to the vector of the length correctors and the radius of the 3 tools $(C)$. Table 1 indicates the inertial tolerances on the workpiece and Table 2 the transition matrix $(A)$ and the correctors towards the deviation of the characteristics. For example, a modification of 1 on the length corrector for tool 1 (CT1L), will increase $c, d$ and $e$ characteristics by 1 , too.

The problem with weighted inertial adjusting is to find the adjusting operations which - once set - will minimise the inertia. In the example proposed, matrix $A$ is not squared, so an infinite number of adjustments are possible.

Inertia is nothing more than the square root of the deviation from the target. The solution to minimise these deviations is, of course, to minimise the least squares. The vector of the corrections is found by calculating:

$$
C=\left[A^{t} A\right]^{-1} A^{t}
$$

The approach consisting in directly applying this relation to the set of measured deviations would, in the case of a stable process, lead to doubling the variance because one would systematically correct any random variance. The use of an inertial control chart $[6,7,11]$ is adapted in order to filter the deviations. This means that only the deviations identified as critical in relation to the inertia or which
Table 3. Filtering by inertial control charts.

\begin{tabular}{ccccc}
\hline $\begin{array}{c}\text { Short term } \\
\text { sigma }\end{array}$ & Value & Deviation & $\begin{array}{c}\text { Filtered } \\
\text { deviation }\end{array}$ \\
\hline 0.0013 & $a$ & 4.997 & -0.003 & 0.000 \\
0.0027 & $b$ & 0.99 & -0.010 & -0.010 \\
0.0027 & $c$ & 1.01 & 0.010 & 0.010 \\
0.0013 & $d$ & 3.008 & 0.008 & 0.008 \\
0.0067 & $e$ & 6.05 & 0.050 & 0.050 \\
\hline
\end{tabular}

Table 4. Proposed corrections.

\begin{tabular}{ccc}
\hline Tool & Corrector & Value of the correction \\
\hline T1 & CT1L length & -0.050 \\
T2 & CT2L length & -0.046 \\
T3 & CT3L length & -0.039 \\
T3 & CT3R radius & -0.003 \\
\hline
\end{tabular}

could be considered as a non-random variation are taken into account.

The example in Table 3 shows some of the values measured on a machining process studied (first workpiece in the series). The deviation on the $a$ characteristic is considered as under control from an inertial point of view; the deviation is therefore considered as equal to 0 .

Then the correction is calculated by equation (2) on the filtered deviations and one finds the values given in Table 4.

\subsection{Weighted multi-criteria inertial adjusting}

Multi-Criteria Inertial Adjusting gives good results when the tolerances are close to each other in value. When certain tolerances are very limited whereas others are much wider in range, an optimisation problem occurs. By minimising the square deviations on the set of characteristics, the adjustment on the most limited tolerances is not optimum.

To overcome this problem, this paper suggests using weighted, multi-criteria inertial adjusting [12], which simply consists in attributing a weighting to matrix $A$ depending on the tolerances of the characteristics concerned. Thus, we bring the set of characteristics to an inertia which is equal to the unit. The optimisation is thus proportional to the inertial tolerance concerned. We minimise the least squares in the unit of standard tolerance. Table 5 shows the weighted values which will favour the adjusting of the most accurate characteristic $(a$ and $d)$.

\section{The problem of optimum adjusting in the case of a small sample lot size}

Weighted Multi-Criteria Inertial Adjusting such as we have just presented it here, is currently being used in many mechanical and aeronautic companies. The results 
Table 5. Value of the weighted corrections.

\begin{tabular}{cccc}
\hline Tool & Corrector & $\begin{array}{c}\text { Value of } \\
\text { the correction }\end{array}$ & $\begin{array}{c}\text { Weighted } \\
\text { correction }\end{array}$ \\
\hline T1 & CT1L length & -0.050 & -0.050 \\
T2 & CT2L length & -0.046 & -0.046 \\
T3 & CT3L length & -0.039 & -0.041 \\
T3 & CT3R radius & -0.003 & -0.001 \\
\hline
\end{tabular}

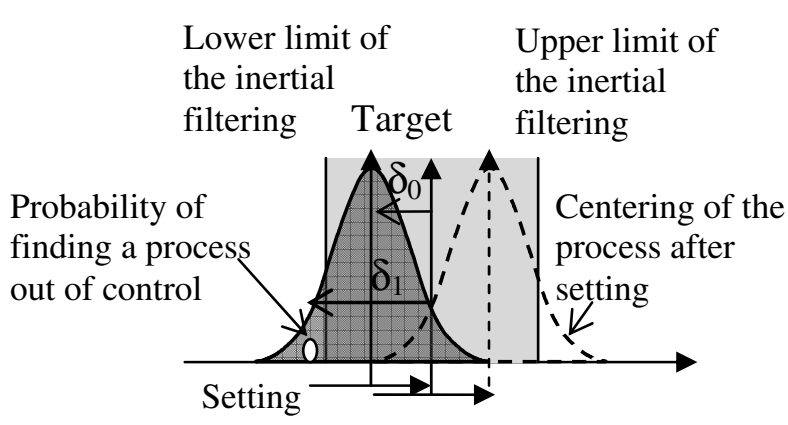

Fig. 2. The problem of determining an adjustment.

show that a real difference in terms of the quality obtained compared to the traditional adjusting methods. The longterm capabilities obtained are often multiplied by three or four. This improvement in the subtlety of the quality of the adjusting operations associated with the automation of the feedback of the measure to the correction of the machine brings other possible points of improvement to the surface. In this article, we will talk about the problem of determining the probable decentring of the process beforehand.

Figure 2 applies to small samples from 1 to $x$ measures. The Gaussian curves represent the average distribution law.

In the first approach, we will consider that the decentring deviation is equal to the measured decentring. This hypothesis sometimes leads to having extra adjusting. Let us illustrate this point with the following example (Fig. 2, hypothesis: the process is stable but off-centre in relation to the target value which is equal to $2 / 3 \mathrm{rds}$ of the filter used).

The decentring is inferior to the limit used to filter the deviation from the target. This limit can be either a limit to the alpha risk, or the limit to the beta risk. However, in the case of small sample sizes, a value can be outside the filtering limits.

In Figure 2, the Gauss bell curve represents either the dispersion of the process in the case of the sample is a unit, or the distribution of the average of $n$ workpieces measured in the case where a sample is used.

The real decentring is equal to $\delta_{0}$.

When a measure is detected outside of the filter, it is introduced into the calculation of the inertial correction with a decentring equal to $\delta_{1}>\delta_{0}$.

The correction made will then decentre the process to the other side of the target, with the major risk of detecting a new situation out of limits which will lead to another correction in the direction from whence it came.

This phenomenon of instability (which is not a major issue in the cases of traditional adjusting) appears a lot more when we carry out multi-criteria inertial adjusting. It prevents one from obtaining process stability such as the long-term capabilities that should be equal to the short term capabilities (process in control).

Even in the case of a perfectly centred process, this phenomenon appears simply because of the false alarms on the $\alpha$ risk on control chart.

Since this risk is multiplied by the number of characteristics monitored, it becomes very big when there are more than ten characteristics. By taking other adjusting rules such as seven points on one side of the average, two points near the limits increase the alpha risk and therefore increase the number of adjusting operations. However, it is not always possible to use the seven-point rule which requires a high frequency of sampling and therefore a prohibitive cost.

\section{Proposition of an adapted adjusting approach}

\subsection{Determining an optimal adjusting}

To put it informally, machine setters often have a tendency to not set the processes from the deviation to the target, but rather a proportion of the deviation. In order to introduce this rule into the algorithms of automatic inertial adjusting, one must be able to determine what the most probable decentring is.

Lill [13] proposed an adjusting rule for the first workpiece manufactured. However, this rule has been shown to be too fixed to be adapted to the different situations encountered during the adjusting of a series. Lill's demonstration considers the following hypotheses. Let us consider the random variable of the measured deviation. This variable is the results from two different elements: a certain disturbance of the adjustment made (unknown) and the dispersion of the population. We consider $\sigma_{S T}$ as the short term standard deviation of the distribution of the population. We also consider that the adjustment is distributed symmetrically around the target with $\sigma_{R}$ as the standard deviation of the distribution of the adjustment errors. If one takes the target value as equal to 0 , the two distribution functions are written as follows:

$$
\begin{aligned}
Y_{P} & =\frac{1}{\sigma_{S T} \sqrt{2 \pi}} e^{-\frac{1}{2}\left(\frac{X_{P}}{\sigma_{S T}}\right)^{2}} \\
\text { and } Y_{R} & =\frac{1}{\sigma_{R} \sqrt{2 \pi}} e^{-\frac{1}{2}\left(\frac{X_{R}}{\sigma_{P}}\right)^{2}} .
\end{aligned}
$$

With:

- $X_{R}$ the adjustment deviation;

- $X_{P}$ the deviation due to random dispersion.

The total error (represented by $E$ ), that is to say the deviation measured by the machine setter, is equal to the 
sum of the two errors. Therefore, we have:

$$
E=X_{R}+X_{P}
$$

In fact, we are looking for the decentring error $\left(X_{R}\right)$, therefore we can write:

$$
X_{P}=E-X_{R}
$$

The measured decentring is a combination of a true decentring and a dispersion. The density of the probability of the measured decentring is calculated by combining two probability laws. This gives us:

$$
Y_{R} Y_{P}=\frac{1}{2 \pi \sigma_{R} \sigma_{S T}} e^{-\left[X_{p}^{2}\left[\frac{\sigma_{R}}{\sigma_{S T}}\right]\right]^{2}+X_{R}^{2}\left[\frac{1}{2} \frac{1}{\sigma_{R}^{2}}\right]}
$$

If we write:

$$
c=\frac{\sigma_{R}}{\sigma_{S T}}
$$

and

$$
Z=X_{P}^{2}\left[\frac{\sigma_{R}}{\sigma_{S T}}\right]^{2}+X_{R}^{2}=c^{2}\left[E-X_{R}\right]^{2}+X_{R}^{2}
$$

The maximum density of the probability of the function $Y_{R} Y_{P}$ is obtained when the $Z$ function is at its minimum. Indeed, the lowest risk of adjusting error will be proportional to the maximum of the density of the probability of the law obtained. By differentiating $Z$ in relation to $X_{R}$, we find:

$$
\frac{\partial Z}{\partial X_{R}}=-2\left[c^{2}\left(E-X_{R}\right)+X_{R}\right]
$$

The distribution function is at its maximum for:

$$
\frac{\partial Z}{\partial X_{R}}=0 \quad \text { or } \quad X_{R}=E \frac{c^{2}}{\left(1+c^{2}\right)}
$$

the best adjustment will be obtained - in the hypotheses that we have retained for our calculations - by an adjustment of the deviation between the dimension measured and the target value $(E)$ multiplied by coefficient $c^{2} /\left(1+c^{2}\right)$.

$$
\text { Optimal Adjustment } A d=E \frac{c^{2}}{\left(1+c^{2}\right)} .
$$

In the case where the measure is done using a sample, the short-term dispersion is such that:

$$
\sigma_{P}=\frac{\sigma_{S T}}{\sqrt{n}} .
$$

In the general case, the optimal adjustment is thus obtained by the relation:

$$
d A=E \frac{n c^{2}}{\left(1+n c^{2}\right)} .
$$

\subsection{Determination of the $c$ ratio}

In the previous equation, the deviation $(E)$ is known. It is the measured deviation, but to determine the optimal adjustment, we must determine the coefficient $c$.

The following hypothesis is put forward for all SPC adjustment: the short-term standard deviation is constant and known. The situation of the decentring is a lot more random and the standard deviation of the adjustment can vary from one situation to another. A given deviation measured is a consequence of a major dispersion in the adjustment.

Therefore we suggest estimating the $c$ coefficient in relation to the ratio between the measured deviation and the short-term standard deviation of the process. Here, the estimation is rather random by taking the rule as being that the ratio is equal to the unit when the deviation is equal to half the dispersion of the process, which represents three short-term standard deviations.

$$
c=1 \quad \text { when } \quad E=3 \sigma_{S T} .
$$

This arbitrary rule is based on the adjusters' common practice which is to adjust to half the measured deviation using the first manufactured workpiece. In the case where $n=1$, the optimal adjustment results in $A d=E / 2$. Then we consider that the $c$ coefficient increases in proportion to the deviation. Indeed, the further we are from the short-term dispersion, the greater the dispersion of adjustment is in relation to the short-term dispersion.

Therefore $c$ is calculated by:

$$
c=\frac{E}{3 \sigma_{S T}} .
$$

Adjustment $A d$ is written as:

$$
A d=E \frac{n E^{2}}{\left(9 \sigma_{S T}^{2}+n E^{2}\right)} .
$$

With

$$
r=\frac{E}{\sigma_{S T}} .
$$

Adjustment $A d$ is written as:

$$
A=\frac{n r^{2}}{\left(9 r+n r^{2}\right)} E .
$$

Term $K=\frac{n r^{2}}{\left(9+n r^{2}\right)}$ represents the proportion of the machines that have put themselves out, and that it is necessary to compensate for depending on the decentring: $\left(r \sigma_{S T}\right)$. This gives the results show Figure 3 .

\subsection{Application using the example in Figure 2}

We will illustrate this approach by basing our application in Figure 2 which used the first manufactured workpiece in the batch (white circle). Let us suppose that the filter in Figure 2 is placed at the three standard deviations alpha risk. The deviation measured is such that: $E=3.2 \sigma_{S T}$. 


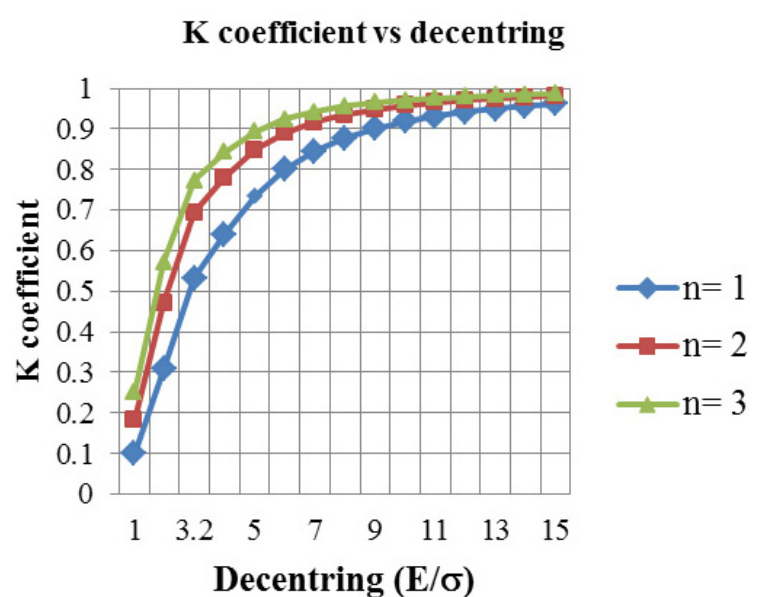

Fig. 3. Determination of $K$ coefficient.

Table 6. Calculation of the $K$ coefficient.

\begin{tabular}{cccc}
\hline$E$ & $n=1$ & $n=2$ & $n=3$ \\
\hline $3.2 \sigma_{S T}$ & $K=0.53$ & $K=0.69$ & $K=0.77$ \\
\hline
\end{tabular}

Depending on the size of the sample, we would find the $K$ coefficient (Tab. 6).

As we are dealing with the first workpiece in the batch, we apply the following formulae: $K=0.53$. In all the cases in the figure, the adjustment converges towards the perfect centring and subsequently avoids generating a decentring on the other side of the target.

\section{Validation by monte carlo simulation}

In order to validate this approach, this part of the paper presents a simulation of a production with a target value of 15 and a short-term standard deviation $\sigma_{S T}=1$. The maximum inertia tolerated will be $I_{\mathrm{Max}}=2$. This allows us to calculate:

$$
C p=\frac{I_{\mathrm{Max}}}{\sigma_{S T}}=2 .
$$

The data are simulated using $10^{6}$ simulations with the data coming from the Mersenne Twister algorithm [14] which generates two random variables $(a$ and $b)$ which have a uniform distribution between 0 and 1 . The Gaussian transformation is done using the following formulae:

$$
y=15+\sqrt{-2 \ln (a)} \cos (2 \pi b) .
$$

Out of 1000000 simulations, we obtained the distribution given in Figure 4. The average is 15, and the standard deviation is 1 .

The $p$-value of the Anderson Darling test (see Fig. 5) is 0.59 , so the normality is accepted. We can notice the excellent distribution on the graph, given by the Mersenne Twister algorithm.

Using this generator of normal law, 8 simulations were carried out, and the following settings were varied:

- the type of process: a stable process or one that has drifted;

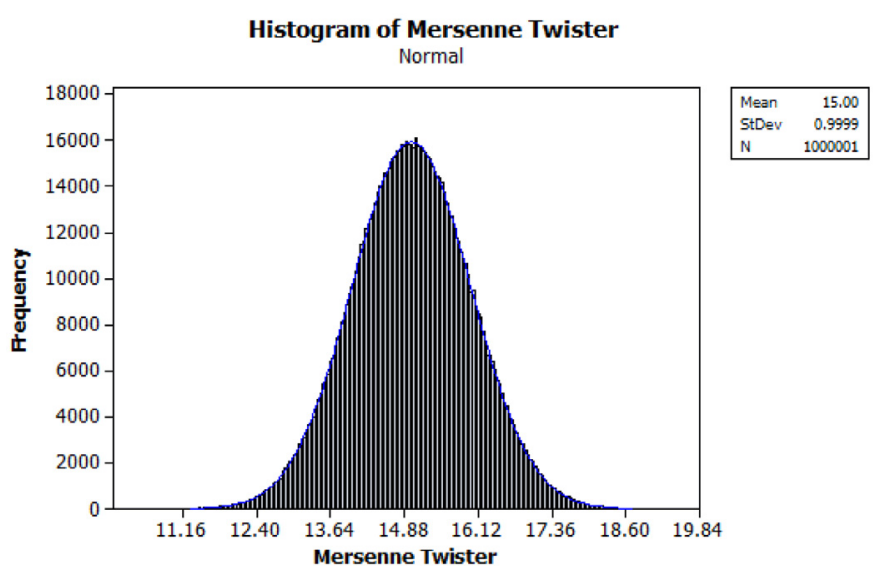

Fig. 4. Histogram of the data provided by the Mersenne Twister.

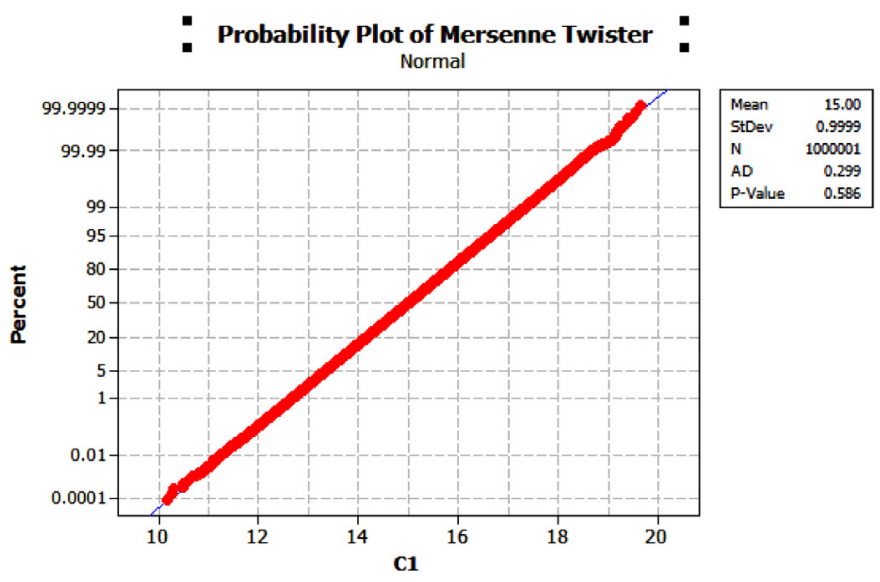

Fig. 5. The Anderson Darling normality test.

- the sample size: $n=1$ and $n=5$;

- the optimisation of the correction : correction of the value of the deviation or optimised correction.

The simulation consists in launching $10^{6}$ simulations. At the beginning, the process is set at the target of: $\delta_{0}=0$. If a value goes out of the Shewhart control limits $(z=3)$, the process is recentred - either by using the deviation between the value measured and the target value - or by the deviation calculated on the most probable decentring $-(R=K E)$.

In this simulation, we suppose that the correction is perfect and that the process moves away from the corrected value.

We have checked that there is no initial decentring that will modify the results. The first decentring is compensated by a false alarm in the 1000 first simulations. In the end, the result on $10^{6}$ simulations is the same.

On the one hand, the results of the simulation give us the number of setups and, on the other hand, the longterm performance $P p, P p i$. The capability indicators are 
Table 7. Results of the simulation.

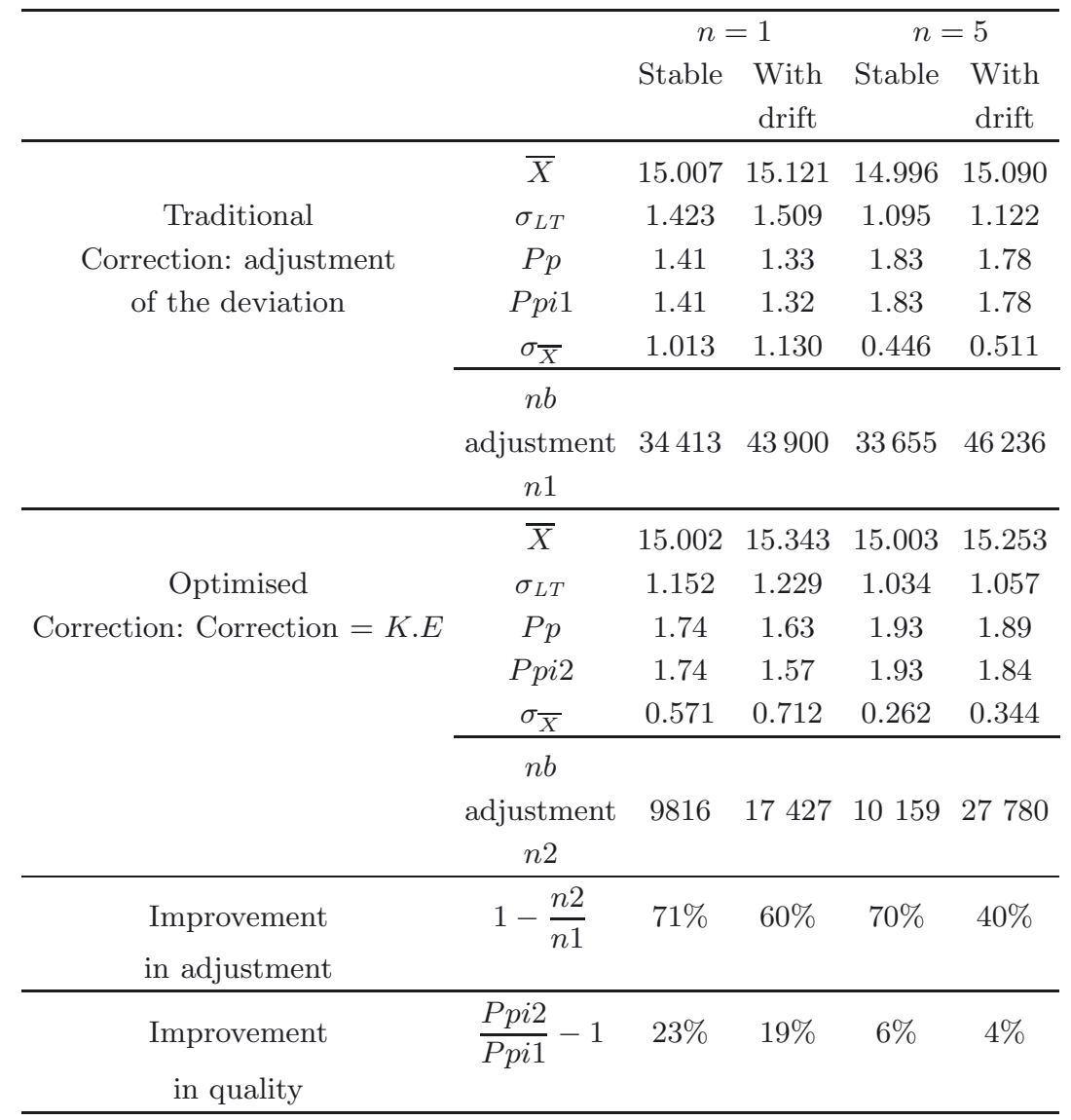

calculated using the relations:

$$
\begin{aligned}
P p & =\frac{I_{\mathrm{Max}}}{\sigma_{L T}}, \\
P p i & =\frac{I_{\mathrm{Max}}}{\sqrt{\sigma_{L T}^{2}+(\bar{X}-\text { Target })^{2}}} .
\end{aligned}
$$

The objective is to obtain a high capability by minimising the number of interventions. The most relevant parameter for quality is the capability index Ppi. Table 7 gives the results of the 8 simulations.

Figures 6 and 7 show the control charts (limited to the first 1000 samples in order for the results on the graphs to be clearly visible).

If we observe the results, we can notice that in all cases, the optimised correction reduced the number of setups (with improvements of up to $70 \%$ ) whilst, at the same time, improving the capability levels obtained. The biggest improvement is obtained when we work with $n=1$ and have a stable production. In the cases with drift, the loss is instant. Table 8 gives the results obtained with $z=4$. We can notice the deterioration of the capability.

The application of the "7 values on the same side of the average" rule generally improves the capability whilst increasing the number of setups.

Table 8 shows the results obtained using the "7 values rule". We can see the deterioration of the capability as well as the improvement reached compared to Table 7 in terms of capability, but with a sharp increase in the number of setups. It is possible to observe that even by applying the "7 values rule", the use of the most probable adjustment remains the most interesting, especially when the process is stable.

\section{Application on a case of setup using an inertial matrix}

In order to illustrate the application of the calculation of the filtering of the adjustment using the coefficient proposed, we will take the example used in Figure 1. The weighted inertial control matrix is given in Table 9 .

From the matrix $A$ (Tab. 2) the weighted matrix $A^{\prime}$ is calculated (Tab. 10) (each row is divided by $6 \times I_{\mathrm{Max}}$ ). Then the control matrix $C^{\prime}$ is calculated by equation (2) (Tab. 9).

By using the previous decentring, the approach proposed leads to the calculation of the probable deviation using the measured deviations and filtered by the inertial chart. The following table (Tab. 11) gives the details of the calculations. If the deviation is lower than $3 \sigma_{S T}$, it is filtered. $c$ is calculated by equation (8), $r$ by equation (10), Ad by relation (11).

The calculation of the correction is made using the matrix product of the probable deviation divided by the 

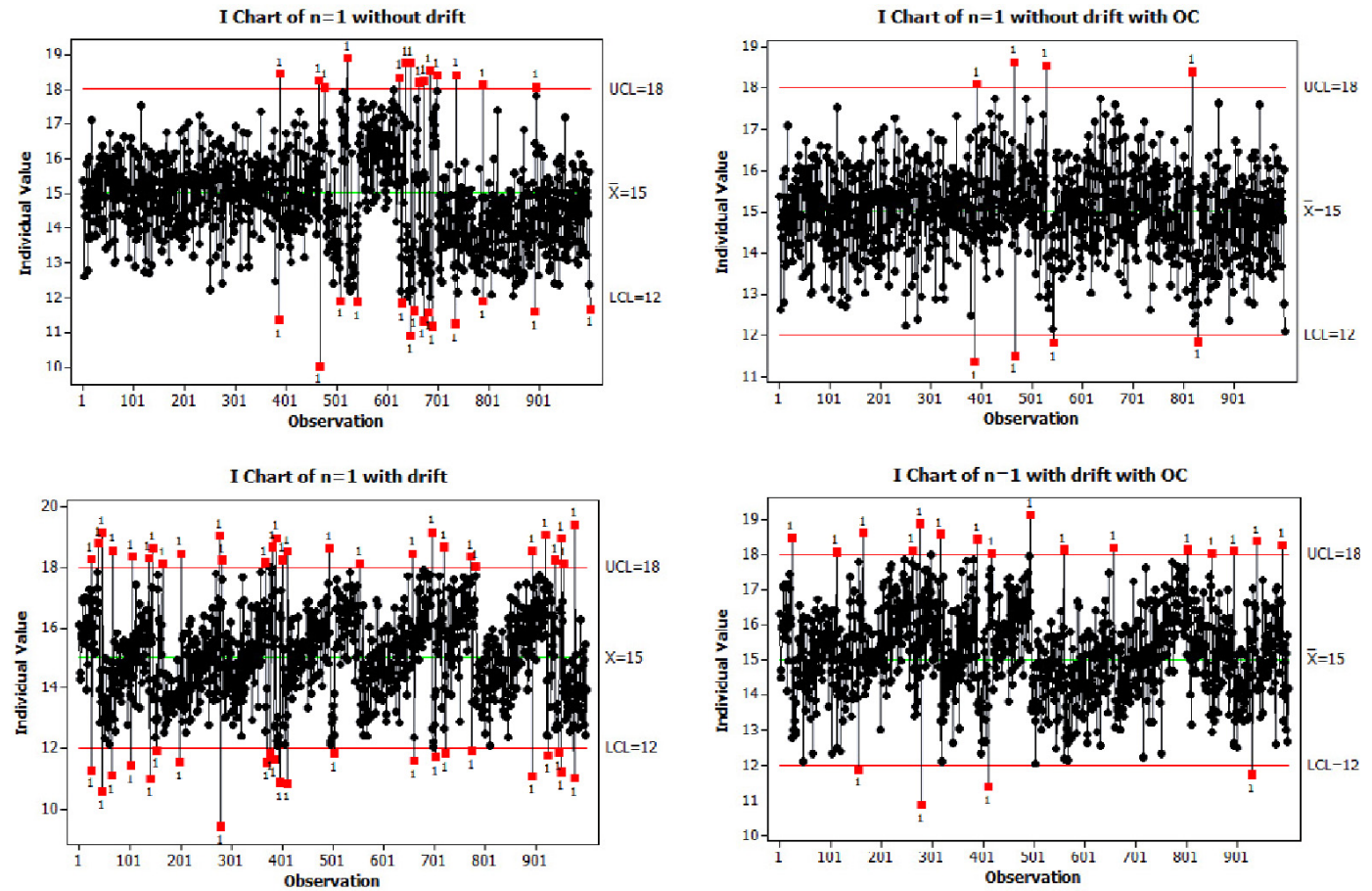

Fig. 6. $n=1$ - Shewhart chart for the first 1000 simulations.
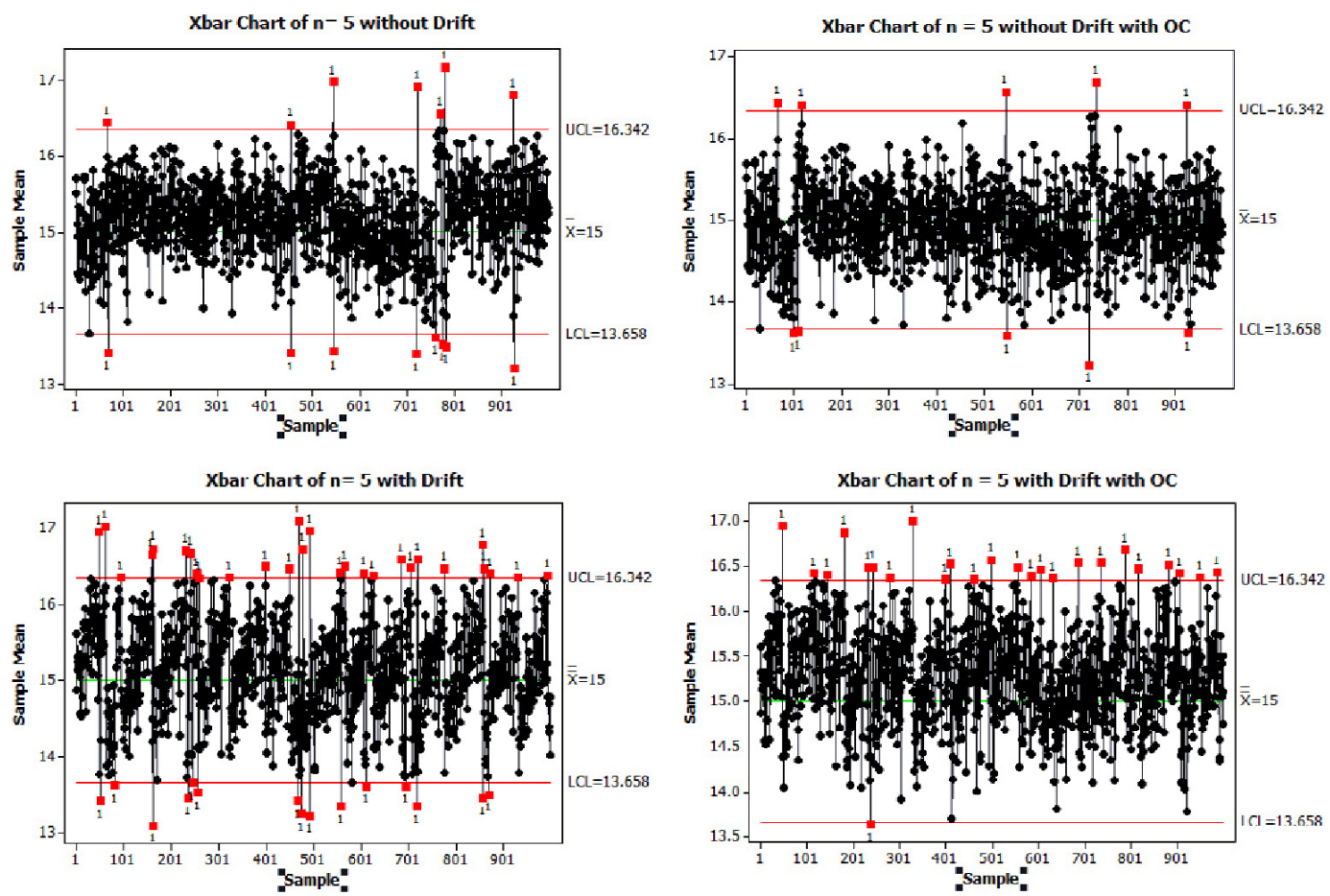

Fig. 7. $n=5$ - Shewhart chart for the first 1000 simulations. 
Table 8. Simulation with $z=4$ and the "7 values rule".

\begin{tabular}{|c|c|c|c|c|c|}
\hline & & \multicolumn{2}{|c|}{$n=1 z=4$} & \multicolumn{2}{|c|}{$n=1, " 7$ value rule" } \\
\hline & & Stable & $\begin{array}{l}\text { With } \\
\text { drift }\end{array}$ & Stable & $\begin{array}{l}\text { With } \\
\text { drift }\end{array}$ \\
\hline \multirow{6}{*}{$\begin{array}{l}\text { Traditional } \\
\text { correction: } \\
\text { Adjustment of } \\
\text { the deviation }\end{array}$} & $\bar{X}$ & 15.227 & 14.879 & 15.001 & 15.190 \\
\hline & $\sigma_{L T}$ & 1.613 & 1.398 & 1.122 & 1.146 \\
\hline & $P p$ & 1.24 & 1.43 & 1.78 & 1.75 \\
\hline & Ppi1 & 1.23 & 1.43 & 1.78 & 1.72 \\
\hline & $\sigma_{\bar{X}}$ & 1.353 & 1.027 & 0.509 & 0.560 \\
\hline & $\begin{array}{c}\text { Number } \\
\text { adjustment } \\
n 1\end{array}$ & 11973 & 5148 & 47150 & 52899 \\
\hline \multirow{6}{*}{$\begin{array}{c}\text { Optimised } \\
\text { correction: } \\
\text { Correction = K.E }\end{array}$} & $\bar{X}$ & 15.652 & 15.112 & 14.998 & 15.298 \\
\hline & $\sigma_{L T}$ & 15.652 & 15.112 & 1.054 & 1.086 \\
\hline & $P p$ & 1.373 & 1.115 & 1.90 & 1.84 \\
\hline & Ppi2 & 1.46 & 1.79 & 1.90 & 1.78 \\
\hline & $\sigma_{\bar{X}}$ & 0.994 & 0.504 & 0.334 & 0.423 \\
\hline & $\begin{array}{c}\text { Number } \\
\text { adjustment } \\
n 2 \\
\end{array}$ & 7392 & 604 & 34596 & 52158 \\
\hline $\begin{array}{l}\text { Improvement } \\
\text { in adjustment }\end{array}$ & $1-\frac{n 2}{n 1}$ & $38 \%$ & $88 \%$ & $26 \%$ & $1 \%$ \\
\hline $\begin{array}{l}\text { Improvement } \\
\text { in quality }\end{array}$ & $\frac{P p i 2}{P p i 1}-1$ & $7 \%$ & $25 \%$ & $7 \%$ & $3 \%$ \\
\hline
\end{tabular}

Table 9. Control Matrix $C^{\prime}$.

\begin{tabular}{lccccc}
\hline & $a$ & $b$ & $c$ & $d$ & $e$ \\
\hline CT1L & 0.00 & 0.00 & 0.00 & 0.00 & 0.10 \\
CT2L & -0.01 & -0.02 & -0.02 & -0.01 & 0.10 \\
CT3L & 0.00 & 0.00 & 0.00 & -0.02 & 0.10 \\
CT3R & -0.02 & 0.00 & 0.00 & 0.00 & 0.00 \\
\hline
\end{tabular}

Table 10. Weighted matrix $A^{\prime}$.

\begin{tabular}{ccccc}
\hline Characteristic & CT1L & CT2L & CT3L & CT3R \\
\hline$a$ & 0.0 & 0.0 & 0.0 & -55.6 \\
$b$ & 0.0 & -23.8 & 23.8 & 0.0 \\
$c$ & 23.8 & -23.8 & 0.0 & 23.8 \\
$d$ & 55.6 & 0.0 & -55.6 & 0.0 \\
$e$ & 9.8 & 0.0 & 0.0 & 0.0 \\
\hline
\end{tabular}

tolerances and the weighted setup matrix.

$$
W F C=C^{\prime} A d^{\prime} .
$$

The weighted vector $A d^{\prime}$ is calculated from the vector $A d$ (each row is multiplied by $6 \times I_{\mathrm{Max}}$ ).

This then gives us the desired adjustments (WFC) that are in Table 12. This example illustrates the proposed calculation well. It reduces the importance of the adjustments to do in proportion to the importance of the decen- tring. It also reduces the number of adjustments significantly.

Today, the experiment carried out over a one year period of batch production has shown that this moderation is very effective. It rarely leads to the necessity of a setup in two stages which would result in an insufficient recentring in order to obtain a perfect centring on the target. Having said that, it eliminates the phenomenon of stratified distribution of products that we emphasised at the beginning of this article. The empirical part of this approach is based on the determining of the situation where the coefficient is fixed at $c=1$. The results obtained seem to show that the choice made here is a suitable one. More hindsight is necessary in order to validate this choice permanently.

\section{Conclusion}

The adjustment of machine-tools in companies in the mechanical industry is still largely carried out in an empirical way by machine setters. The weighted, multi-criteria inertial piloting that we looked at briefly at the beginning of the article, already offers a considerable improvement in terms of the quality of the products manufactured. However, a phenomenon of stratified distribution remained within the weighted inertial approach, due to over-adjustment and unsuitable settings. This leads to a severe drop in capability between the short-term and the long-term. 
Table 11. Proposed corrections.

\begin{tabular}{ccccccccc}
\hline $\begin{array}{c}\text { ST } \\
\text { sigma }\end{array}$ & Value & Deviation & $\begin{array}{c}\text { Filtered } \\
\text { deviation }\end{array}$ & $c$ & $r$ & $K$ & $\begin{array}{c}\text { Probable } \\
\text { deviation } A d\end{array}$ \\
\hline 0.0013 & $a$ & 4.997 & -0.003 & 0.000 & 0 & 0 & 0.000 & 0.000 \\
0.0027 & $b$ & 0.99 & -0.010 & -0.010 & 1.25 & -3.75 & 0.610 & -0.006 \\
0.0027 & $c$ & 1.01 & 0.010 & 0.010 & 1.25 & 3.75 & 0.610 & 0.006 \\
0.0013 & $d$ & 3.008 & 0.008 & 0.008 & 2 & 6 & 0.800 & 0.006 \\
0.0067 & $e$ & 6.05 & 0.050 & 0.050 & 2.5 & 7.5 & 0.862 & 0.043 \\
\hline
\end{tabular}

Table 12. Proposed corrections.

\begin{tabular}{ccccc}
\hline Tool & Corrector & $\begin{array}{c}\text { Value of } \\
\text { the correction }\end{array}$ & $\begin{array}{c}\text { Weighted } \\
\text { correction }\end{array}$ & $\begin{array}{c}\text { Weighted } \\
\text { and filtered } \\
\text { correction (WFC) }\end{array}$ \\
\hline T1 & CT1L & -0.050 & -0.050 & -0.043 \\
T2 & CT2L & -0.046 & -0.046 & -0.040 \\
T3 & CT3L & -0.039 & -0.041 & -0.036 \\
T3 & CT3R & -0.003 & -0.001 & -0.001 \\
\hline
\end{tabular}

In this article, we have proposed an approach which allows the calculation of a probable decentring depending on the decentring observed and the short-term dispersion of the process. This theoretical demonstration has been validated by a Monte-Carlo simulation which shows the gains obtained by the approach as much in terms of the number of adjustments necessary as in the quality of the production obtained.

Future research will allow us to more precisely determine the $c$ coefficient especially using the feedback on experiences that we could have in real piloting situations.

Looking back over 6 months of its application in industry, we can show that by using this probable decentering instead of estimated decentering by the measure of a sample, we obtain results which eliminate the phenomenon of stratified distribution. In addition, the quality obtained using the machine-tools piloted in this way also meets the level of quality required today.

\section{References}

1. M. Pillet, F. Bernard, L. Avrillon, Le tolérancement inertiel, une autre façon d'intégrer l'aspect combinatoire dans les processus assemblés, Congrès CPI 2001, CD-ROM (Fès, Morocco, 2001)

2. M. Pillet, A. Sergent, D. Duret, Weighted inertial tolerancing, $5^{\text {e }}$ Congrès Int. Pluridisciplinaire Qualité et Sûreté de Fonctionnement (Qualita 2003, Nancy, France, 2003), pp. $111-118$

3. M. Pillet, Inertial Tolerancing, The Total Quality Management Magazine (2004), Vol. 16, pp. 202-209

4. M. Pillet, S. Samper, F. Formosa, Geometrical Inertial Tolerancing, $6^{e}$ Congrès Int. Pluridisciplinaire Qualité et Sûreté de Fonctionnement (Qualita 2005) (Bordeaux, France, 2005), pp. 757-764
5. M. Pillet, D. Denimal, P.A. Adragna, S. Samper, Interest of the inertial tolerancing method in the case of watchmaking micro assembly, Micro-Assembly Technologies and Applications, IFIP (2008), Vol. 260, 189-197

6. D. Denimal, M. Pillet, A. Sergent, Détermination des paramètres d'une carte de contrôle dans un contexte de tolérancement inertiel, Qualita 2009 (Besançon, 2009)

7. M. Pillet, P.A. Adragna, V. Ozouf, A.S. Guerra, Pilotage par carte de contrôle dans le cas d'un tolérancement inertiel, Qualita 2007 (Tanger, Maroc, 2007)

8. E. Goldschmidt, E. Pairel, M. Giordano, M. Pillet, Changeover from the functional geometric tolerances to the dimensional parameters to be supervised in production, CIRP J. Manuf. Syst. 36, 305-309 (2007)

9. E. Goldschmidt, E. Pairel, Détermination systématique des étapes de contrôle et des cotes de fabrication pour les procédés d'usinage, $18^{e}$ Congrès Français de Mécanique (France, 2007)

10. E. Pairel, E. Goldschmidt, P.A. Adragna, P. Hernandez, M. Pi, The pilot dimensions method: Reconciling Steering and Conformity in Workshops, Int. J. Product. Res. 1-14 (2010)

11. D. Denimal, M. Pillet, A. Sergent, Inertial Control Chart, Industrial Application and feedback, IDMMEVirtual Concept (Beijing, China, 2008)

12. M. Pillet, Améliorer la productivité, déploiement industriel du tolérancement inertiel, edition d'organisation (2010), ISBN 978-2-212-54754-222

13. H. Lill, Y. Chu, K. Chung, Statistical Set-up Adjustement for low volume manufacturing, Statistical Process Control in Manufacturing (Dekker, 1991), pp. 23-38

14. M. Matsumoto, T. Nishimura, Mersenne twister: a 623-dimensionally equidistributed uniform pseudo-random number generato, ACM Transactions on Modeling and Computer Simulation (TOMACS), Special issue on uniform random number generation (TOMACS Homepage archive, 1998), Vol. 8 